\title{
Africa Checklist of: Families, Genera, and Species of Ectoparasitic Mites Found in the Palaearctic and Afrotropical Regions
}

\author{
Kawther M. EI Kammah and Hanan S. Gabr \\ Zoology Department, Faculty of Agriculture, Cairo University,Giza,Egypt.
}

\begin{abstract}
This Checklist includes 23 families, 74 Genera, 281 species of ectoparasitic mites distributed in 23 countries in Africa throughout the Palaearetic and Afrotropical regions. Data collected from Cameroons was the highest (20.5\%), followed by S. Africa (17.5\%), Egypt (14.5\%). Data collected ranged from 0.5 to $5.0 \%$ from each of the other 20 countries included. Parasitic mites on birds belong to 14 families ( 7 infest skin and 7 feather mites). Their hosts belong to: Class: Aves:- Order: Anseriformes (duck, fowl, turkey...etc.). Columbiformes (pigeon, dove ...etc.), Passeriformes (canary, sparrows...etc.). Parasitic mites on animals belong to 9 families (4 mycoptic mange, 2 itch mites, one hair follicle mange and 2 skin tissue mites).Their hosts belong to: Class: Mammalia: Order: Carnivora (dogs, cats,...etc.), Perissodactyla-(horses...etc.), Artiosdactyla (sheep, pig, goats...etc.), Lagomorpha (rabbits...etc.), Rodentia, (rodents...etc), Primates (man...etc).
\end{abstract}

Key Words: Africa, Palaearetic, Afrotropical, Acari, Ectoparasitic mites.

Acari is the only group among Chelicerata which includes plant and animal parasites. Almost all animals larger than mites are parasitized by them. However, worldwide, parasitic mites are one of the least studied groups of animals despite their medical and economic importance, Baker \& Wharton (1956), Baker et al. (1956), El Kammah et al. (1982a, b \& 2007) and Hoogstraal (1984). Knowledge of mite species in the African fauna is still relatively sparse and few species are studied, Atyo \& Guad (1970 \& 1971); Nutting \& Desch (1972), and Krantz (1978).

Special attention was directed to mite studies in Egypt, Kenya and Sudan by Keegan (1956) and in S. Africa by Zumpt (1961). They covered most African regions, (Africa Tropic of Cancer including Sahara). These regions were recently renamed by Crosskey \& White (1977) and Kettle (1995) as the Palaearetic : (Africa north of the Tropic of Cancer including Sahara) and Afrotropical regions : (All Africa south of the Tropic of Cancer excluding the Sahara).

However, nomenclature of most species has been subject to change. It is our target to establish the present nomenclature of ectoparasitic mites found in different countries in Africa, (Fig.1). Information about each species listed herein includes synonymy, host and distribution, as compiled from previously published research work.

\section{Family: Analgidae (Trouessart \& Megnin) \\ -Subfamily Analgesinae \\ 1- Anhemialges (Gaud) \\ An. longipes (Trouessart) \\ Host: Birds. \\ 2-Anlges (Nitzsch)}

A. cercodons (Gaud and Mouchet) A. corvinus

(Megnin), A. eurygaster (Gaud and Mouchet)
A. leiopus (Gaud and Mouchet), A. unidertatus (Berlese).

\section{3-Berlesella (Trouessart)} Ber. aestilvalis (Berlese).

\section{4-Hemialges (Trouessart)}

Hem. spinosus (Trouessart).

5-Heteralges (Gaud and Mouchet)

Het. ostracopus (Gaud and Mouchet).

Host: Birds.

Distribution: Cameroons.

\section{6- Megninia (Berlese)}

M. columbae (Boscholz), M. Cubitalis (Megnin),

$M$. ginglymura (Megnin)

Host: Chicken, ducks, pigeons, turkey.

Distribution: Egypt.

- Subfamily: Psoroptoinae

Psoroptoides(Trouessart),

P. cryptonychus (Gaud and Mouchet).

P. haplonychus, (Gaud and Mouchet).

P. uroplax (Gaud and Mouchet).

Host: Birds.

Distribution: Cameroons.

Family: Avenzoariidae (Oudemans)

Bdellorlynchus(Megnin and Trouessart)

B. polymorphus (Trouessart).

Host: Ducks

Distribution: Egypt.

B. psalidurus (Trouessart).

Host: Ducks, geese.

Distribution: Cameroons, Congo.

Family: Demodecidae (Nicolet)

Demodex (Owen)

D. bovis (Stiles), D. canis (Leydig),

D. caprae (Railliet), D. folliculorum (Simon), 


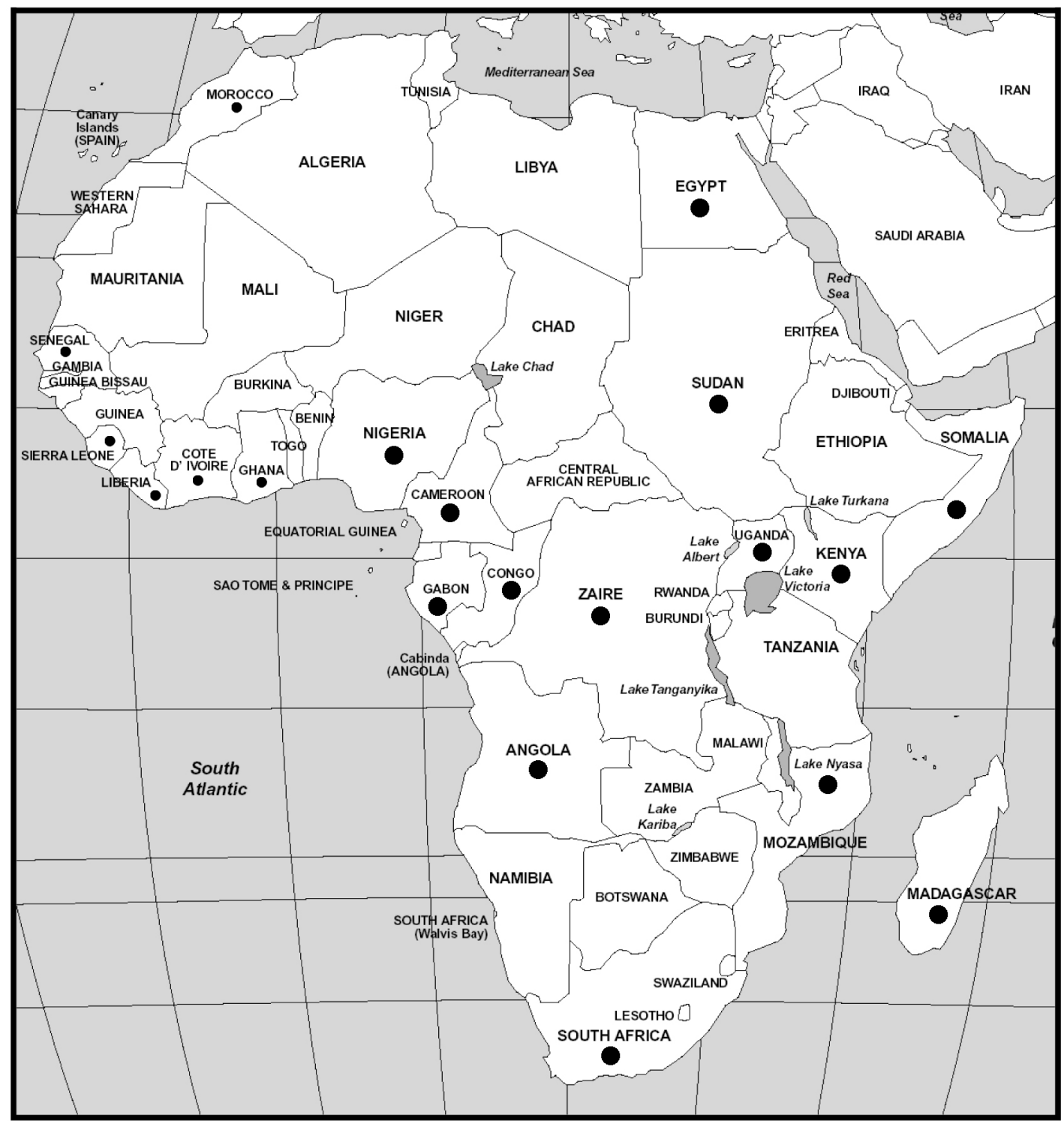

Fig. (1): The distribution of ectoparasitic mites in different countries in Africa.

D. ovis (Railliet), D. phylloides (Csokor), D. suis (Railliet), D. privis.

Host: Cattle, dogs, goats, man, pigs and sheep.

Distribution: Egypt, S. Africa, (and throughout Afrotropical Region).

\section{Family: Dermanyssidae (Kolenati)}

Subfamily: Dermanyssinae

1-Dermanyssus (Duges)

D. gallinae (De Geer).

Host: Fowl, canary, Birds.

D. muris (Hirst).

Host: rats.

Distribution: Egypt, S. Africa.

\section{2-Allodermanyssus (Ewing)}

A.aegyptius (Hirst), A. sanguineus (Hirst).

Host: Rats, mice.

Distribution: Egypt, Sudan.
Family: Dermoglyphidae (Vitzthum)

Subfamily: Dermoglyphinae

1- Dermoglyphus (Megnin)

D. columbae (Sugimotos).

Host: Pigeons, sparrows.

Distribution: Egypt.

D. diplectrum (Trouessart).

Host: Geese.

Distribution: Cameroons.

D. elongatus (Megnin).

Host: Fowl.

Distribution: Cameroons.

D. pachycnemis (Trouessart).

Host: Ostrich.

Distribution: S. Africa.

2- Paralgoides (Gaud and Mouchet)

P. anoplopus (Gaud and Mouchet). 
Host: Birds.

Distribution: Cameroons.

P. deformis (Trouessart and Neumann).

Host: Birds.

Distribution: Angola.

P. nototrchus (Gaud and Mouchet).

Host: Fowl.

Distribution: Cameroons.

Subfamily: Falculiferinae

1- Falculifer (Railliet)

F. cameronensis (Gaud and Mouchet).

Host: Doves.

Distribution: Cameroons.

F. rostratus (Buchholz).

Host: Doves, pigeons.

Distribution:Cameroons,S.Africa, Sudan.

2- Pterophagoides (Gaud and Mouchet)

P. bathmourus (Gaud and Mouchet).

Host: Doves, pigeons.

Distribution: Cameroons.

3- Pterophagus (Megnin)

P. brachysoma (Gaud and Mouchet),

P. Monacrotrichus (Gaud and Mouchet),

P. similis (Gaud and Mouchet).

Host: Doves.

Distribution: Cameroons.

P. strictus ( Megnin) .

Host: Pigeons.

Distribution: S. Africa.

4- Rhipirus (Gaud and Mouchet)

$R$. copephorus (Gaud and Mouchet).

Host: Pigeons.

Distribution: Cameroons.

Family: Epidermoptidae (Trouessart)

1- Dermation (Trouessart and Neumann)

D.bihamatum (Trouessart and Neumann)

2- Epidermoptes (Rivolt)

E. bilobatus (Rivolt).

Host: Fowl, birds.

Distribution: S. Africa.

3- Yunkeracarus (Fain)

Y.muris (Fain).

Host: Rats.

Distribution: Brundi, Ruanda.

Family: Freyanidae (Dubinin)

1- Freyana (Haller)

$F$. anatina (Koch).

Host: Ducks, Geese.

Distribution: Cameroons.

F.largifolia (Megnin\&Trouessart).

Host: Ducks, geese.

Distribution: Egypt, S. Africa, Sengal.
2- Feryanomorpha (Gaud)

Fer.ambigua(Gaud), Fer. polymorpha (Gaud).

Fer. diversa (Gaud).

Host: Birds.

Distribution: Cameroons.

3-Hemifreyana (Gaud and Mouchet)

H. leclerci (Trouessart).

Host: Birds.

Distribution: Nigeria and Cameroons.

H. marginata (Trouessart).

Host: Birds.

Distribution: Congo, Cameroons.

Family: Harpyrhynchidae(Megnin),

1-Harpyrhynchus (Megnin),

H. crista-galli (Berlese and Trouessart)

Host: Birds

Distribution: Kenya, S. Africa, Tanzania and

Uganda.

H. pectinifer (Lawrence), H. pilirostris (Berlese and Trouessart).

Host: Birds, Sparrows.

Distribution: S. Africa.

Family: knemidocoptidae (Furstenberg)

Knemidocoptes (Furstenberg)

$K$. mutance (Robin and Lanquetine)

Host: Chicken, turkey.

Distribution: Egypt.

K. pilae (Lavoipierre and Griffiths)

Host: Canaries.

Distribution: S. Africa.

Family: Laelapidae (Berlese)

Subfamily: Haemogamasinae

1-Davisiella (Zumpt and Patterson),

D. reticulata (Zumpt and Patterson).

Host: Rodents.

Distribution: Zimbabui.

2-Haemogamasus (Berlese)

H. liberiensis (Hirst).

Host: Rodent nests.

Distribution: Kenya.

3- Haemolaelaps (Berlese)

$H$. bathyergus (Radford), H. callosus (Berlese),

$H$. capenssis (Hirst), H. casalis (Berlese),

H. centrocarpus (Berlese), H. cryptomius

(Radford), H. dasymys (Radford), $H$. davisi

(Zumpt and Till), H. eloffi (Zumpt and Till),

H. galagus (Lavoipierre), H. glasgowi (Ewing),

$H$. haydocki (Till), H. hirsti (Keegan), H. hystrici

(Zumpt and Till), H. inpos (Berelse),

H. labuschagnei (Zumpt and Patterson),

$H$. lophuromius (Radford), $H$. mesopicos

(Radford), H. murinus (Berelse), H. radfordi 
(Keegan), H. setyni (Till), H. sudanicus (Zumpt and Till),H. tachyoryctes ( Radford), H. taterae (Radford), $H$. tateroins (Radford), H. villosissimus (Berelse).

Host: Birds, dogs, gerbils, mice, rats,. spiny rats.

Distribution: Cameroons, Congo, Kenya,

Mozambique Nigeria., S. Africa Somaliand, Sudan,Uganda and Zimbabwe.

\section{Subfamily -Laelapinae \\ 1- Andreacarus (Radford), A.petersi (Radford), A. zumpti (Tufflieb) \\ Host: Giant rats. \\ Distribution: Cameroons, Congo.}

\section{2- Androlaelaps Berelse .}

A. arvicanthis (Radford), A. equatoriensis (Keegan), A. graingei (Zumptand Patterson), A. lehfeni (Oyoun and ElKady). A. marshalli (Berlese), A. zudemansi A. oudemansi. (Radford), A.zuluensis (Zumpt).

Host: Rodents, rats, gerbils.

A. casalis (Berlese), A. zaheri (Hafez, El Badry \& Nasr).

Host: Chicken, ducks.

Distribution: Egypt, Kenya, Sudan, Uganda

\section{3- Hypoaspis (Canestrini)}

H. orientalis (Hafez, El Badry \& Nasr).

Host: Chicken farms.

Distribution: Egypt

4- Laelaps (Koch)

L.aethiopicus (Hirst), L. brandbergensis (Taufflieb), L. Congoicola (Berlese), L. echidninuss (Berlese), L. Garndis (Hirst), L. giganteus (Berlese), L. grenieri (Taufflieb), L. Kamplensis, L. lamporni (Hirst), L. lavieri (Taufflieb), L. lavoipierrei (Taufflieb) L. liberinsis (Hirst), L. longiventris (De Meillon and Lavoipierre), L moucheti (Taufflieb), L. muricola (Tragardh) L. nuttalli (Hirst), $L$ parvulus (Hirst), L. peregrinus (Taufflieb), L. praomyia (Taufflieb), L. roubaudi (Taufflieb), L. simillimus (Zumpt), L. sinai (El Kammah, Oyoun \& El Kady), L. spinifer (Taufflieb and Mouchet), L. thamnomys (Taufflieb), L. tillae (Taufflieb), L. transvaalensis (Zumpt), L. ugandanus (Hirst), L. vansomereni (Hirst), L. wittei (Cooreman), L. yaoundensis (Taufflieb and Mouchet), L. zumpti (Keegan).

Host: Shrew, rodents, rats, mice.

Distribution:Egypt, Cameroons, Congo, Ivory coast., Kenya,Liberia, Madagascar, S. Africa, Sudan, Uganda, Zimbabwe.

\section{5- Radfordialelaps (Zumpt)}

R. meridonalis (Zumpt).
Host: wild rabbits.

Distribution: S. Africa.

6- Ugandolaelaps (Radford)

U. protoxera.

Host: Squirrel.

Distribution: Cameroons, Uganda.

Family: Listrophoridae (Canestrini)

Subfamily: Atopomelinae

1-Chirodiscoides (Hirst)

C. caviae (Hirst).

Host: Guinea pigs.

Distribution: S. Africa.

\section{2-Listrophoides (Hirst)}

L. aethiopicus (Hirst). L. africanus (Radford).

L. dasyms (Radford). L. expansus (Ferris).

L. lemniscomys (Radford).. L. mastomys

(Radford), L. womersleyi (Lawrence).

Host: Rats.

Distribution: S. Africa. Uganda, Congo, Sierra Leone.

\section{Subfamily: Listrophorinae}

Listrophorus (Pagenstecher).

L.arishi (Oyoun, Kammah and El Kady).

L. bothae (Hirst).

Host:Gerbils.

Distribution: Congo, Egypt, S. Africa, Uganda.

L.gibbus(Pagenstecher).

Host: Goats, rabbits, sheep.

Distribution: Egypt, S. Africa.

L. lophuromys (Radford).

Host: Rat.

Distribution: Sierra Leone

Family: Macronyssidae (Oudemans)

1- Astridiells (Fain)

A.capitatus(Strandtmann),A.caprimulgi.

Host: Birds.

Distribution: Ruanda.

\section{2-Macronyssus (Kolenati)}

M.bacoti(Hirst), M. dendropicos (Radford),

M. sangsteri (Radford).

Host: Birds, mice, squirrel, rats.

M. bursa (Berlese), M. sylviarum (Canestrini and Fanzago)

Host: Fowl.

Distribution: Cameroons, Egypt, Kenya, S. Africa, Uganda.

\section{3- Ornithonyssus (Sambon)}

O. aethiopic (Hirst), O. bacati (Hirst).

Host: Rabbits, rodents.

O. bursa (Berlese).

Host: Chickens. 
Distribution: Egypt.

4- Pellonyssus (Clark and Yunker)

P. biscutatus (Hirst), P.reedi (Zumpt and Patterson), P. similis (Zumpt and Till).

Host: Birds, sparrow.

Distribution: Mozambique, S. Africa.

5- Raillietia (Trouessart)

R.auris(Leidy).

Host: Cattle.

Distribution: Central Africa.

6- Ruandanyssus (Fain)

R.buboensis (Fain).

Host:Owls. Distribution: Mozambique

Family: Myialgesidae (Dubinin)

1- Microlichus (Trouessart)

M.avus (Trouessart)

Host: Birds.

Distribution: S. Africa.

2- Myialges (Sergent and Trouessart) M. anchora (Sergent and Trouessart).

Host: Pigeon.

Distribution: S. Africa.

Family: Myocoptidae (Megnin)

1- Mycoptes (Claparede)

M. musculinus (Koch).

Host: Rodents.

Distribution: Egypt, S. Africa.

2- Neomycoptes (Lawrence)

N. tenax (Michael).

Host: Gerbil.

Distribution: (Throughout the Afrotrpical region).

Family: Myobiidae (Megnin)

1- Afromyobia (Radford)

A. trouessarti (Poppe).ero

Host: Shrew.

Distribution: Congo.

2- Myobia (Von Heyden)

M. muris-musculi (Schrank),

M. otomyia (Lawrence).

Host: Mice, rats.

Distribution: S. Africa.

3- Radfordia (Ewing)

R. affinis (Poppe), R. ensifera (Poppe).

Host: Mice, rats.

R. forcipifer (Lawrence).

Host: Gerbils. $R$. rotundata (Lawrence).

Host: Mole rats.

Distribution: S. Africa.
Family: Proctophyllodidae (Magnin \& Troussart)

Subfamily: Alloptinae

1- Brephosceles (Hull)

B. charadii (Dubinin), B. decapus (Gaud),

$B$. discosurus (Troussart), B. dolichocaulus

(Gaud and Mouchet),

Host: Birds.

Distribution: Cameroons, Sengal.

B. gynurus (Troussart).

Host: Geese.

Distribution: Congo.

B. histionotus (Gaud and Mouchet),

B. vanelli (Gaud).

Host: Birds.

Distribution: Cameroons, Morocco.

2- Thysanocercus (Gaud and Mouchet)

T. callithyrus (Gaud and Mouchet),

T. cypseli (Canesrini and Berlese).

Host: Birds.

Distribution: Cameroons.

Subfamily: Proctophyllodinae

1- Anisodiscus (Gaud and Mouchet)

A. dolichogaster (Gaud), A. megadiscus

(Gaud and Mouchet).

Host: Birds.

Distribution: Cameroons.

A. eupariphus (Gaud and Mouchet).

A.megaeaulus (Troussart).

Host: Birds

Distribution: Sengal, Cameroons.

2- Proctophyllodes (Robin)

P. acanthicaulus, P. doleophyes (Gaud).

Host: Birds.

Distribution: Morocco and Cameroons.

P. africanus (Gaud).

Host: Sparrows.

Distribution: Sudan.

P. anisogamus (Gaud and Mouchet).

Host: Fowl.

Distribution: Cameroons.

P. orientalis (Gaud).

Host: Sparrows.

Distribution: Egypt.

P. stericlus (Megnin).

Host: Turkey.

Distribution: Egypt.

\section{Subfamily Pterodectinae}

1- Pterodectes (Robin)

P. listroproctus (Gaud and Mouchet).

Host: Fowl. 
Distribution: Cameroons.

P. merulae (Gaud).

Host: Birds.

Distribution: Cameroons, Morocco.

P. ranci (Gaud), P. platynocercus (Gaud andMouchet).

Host: Birds.

Distribution: Cameroona, Madagascar.

P.rhodesiensis (Till).

Host: Birds.

Distribution: Mozambique, Zimbabwe

P. rutilus (Robin).

Host: Birds.

Distribution: Cameroons.

Subfamily: Trouessartiinae

1- Allanalges (Trouessart)

A. leiophyllus (Gaud and Mouchet)

Host: Birds.

Distribution: Cameroons, Sengal.

2- Trouessartia (Canestrini)

T. africana (Till).

Host: Birds.

Distribution: Zimbabwe

$T$ incisa (Gaud). T. simillima (Gaud).

Host: Birds.

Distribution: Morocco, Cameroons

Family: Psoroptidae (Canestrini)

1- Chorioptes (Gervais and Beneden)

Ch. bovis (Hering). Ch.ovis (Gerlach).

Host:Goats, rabbits, sheep.

Distribution: Egypt, S. Africa.

Ch.crewei (Lavoipierre).

Host: Cattle.

Distribution: Cameroons.

2- Otodectes (Canestrini)

O. cynotis (Hering).

Host: Cats, dogs.

Distribution: Egypt, S. Africa.

\section{3- Psoroptes (Gervais)}

P. equi (Hering), P. cuniculi (Delafond),

P. ovis (Womersley).

Host: Cattle, goats, horses, rabbits, sheep.

P. natalinsis (Hirst).

Host: Buffaloes, cattle.

Distribution: Egypt, S. Africa.

Family: Pyroglyphidae (Cunliffe)

1-Dermatophagoide (Bogdanow)

D. farinae (Hughes\&Seoidi)

D. pteronyssinus (Trouessart \& Seoidi)
Host: Chickens.

Distribution: Egypt.

2- Pyroglyphus

P. africanus (Hughes).

Host: Chicken.

Distribution: Egypt.

Family: Rhinonyssidae (Trouessart)

1 - Larinyssus (Strandtmann)

L. orbicularis (Strandtmann).

Host: Birds.

Distribution: Brundi, Ruanda.

2- Neonyssus (Hirst)

N. beloplskii (Bergetova).

Host: Herons.

N. bubulci (Zumt and Till).

Host: Cattle, birds.

N.buteonis (Fain).

Host: Egles, birds.

N.columbae(Crossley), N.smelloi Castro).

Host: Pigeon.

N.treronis (Fain).

Host: Birds.

Distribution: Brundi, Ruanda, S. Africa.

\section{3- Rhinonoyssus (Trouesart)}

$R$. afribyx (Fain), R. apus (Fain), R. himantopus (Strandtmann), R. oliocephali (Fain).

Host: Birds.

R. rhinolethrum (Trouessart).

Host: Ducks, geese.

Distribution: Brundi, Ruanda, S. Africa.

\section{4- Sternostoma (Berlese and Trouessart)}

$S$. boydi (Strandtmann). S. colli (Fain). $S$. cooremani (Fain). S. cuculorum (Fain). S. dureni (Fain), S. lanoirum (Fain). S. nectarinia (Fain), $S$. Sturnicola (Fain).

Host: Birds.

Distribution: Brundi, Ruanda.

S. thienponti (Fain).

Host: Birds.

Distribution: Brundi, Ruanda.

Family: Sarcoptidae (Trouessart)

Subfamily: Sarcoptinae (Murray)

1- Notoedres (Railliet)

$N$. (N.) cait (Hering).

Host: cats.

Distribution: S. Africa

$N$. (N.) muris (Megnini).

Host: Rodents.

Distribution: Guinea, S. Africa. 
$N$. (N.) oudemansi (Fain).

Host: Hedgehog, rats.

Distribution: Kenya Ruanda.

$N$. (N.) pahangi.

Host: Rodents.

Distribution: Kenya, S. Africa.

$N$. (N.) pseudomuris (Lavoipierre).

Host: Mice.

Distribution: Egypt.

2- Prosarcoptes (Lavoipierre)

$P$. pitheci (Philippe).

Host: Monkeys.

Distribution: Guinea.

3-Sarcoptes (Latereille)

S. bovis (Cameron).

Host: Cattle.

Distribution: S. Africa.

S. canis (Gerlach).

Host: Dogs, man

S. caprae (Furstenberg).

Host: Goats.

S. equi (Gerlach).

Host: Horses.

S. megnini (Bedford).

Host: Sheep.

S. ovis (Bedford).

Host: Rabbits, sheep.

Distribution: Egypt.

S. precox (Canestrini).

Host: Rabbits

S. scabiei (Linnaeus).

Host: Man, rodents, wolf.

Distribution: Egypt.

S. suis (Gerlach).

Host: Pigs.

Distribution: S. Africa.

4-Trixacarus (Sellnick)

T. eliurus.

Host: Rodents.

Distribution: Madagascar.

Family: Syringophillidae (Trouessart)

Syringophilus (Heller)

S. columbae (Hirst)

Host: Fowl, pigeons.

Distribution: Egypt, S. Africa.

S. zumpti (Lawrence).

Host: Birds.

Distribution: (Throughout Afrotrpical region).
Family: Trombiculidae (Ewing)

Subfamily: Trombiculinae

Trombicula (Berles)

T. holosericeeum.Host: Cattle, dogs, rodents.

Distribution: Egypt.

T. jadini (Vercammen- Grandjean),

T. panieri (Jadin and Vercammen-Grandjean), $T$.

Rodhaini (Jadin and Vercammen- Grandjean).

Host: Rodents.

Distribution:Brundi,Cameroons, Ruanda.

T. mastomyia (Raford).

Host: Rats, fowl.

Distribution: Central Africa.

T. mini (Vercammen- Grandjean and Brennan),

T. rossi (Vercammen- Grandjean and Brennan).

Host: Rodents.

Distribution: Uganda.

T. bruynoghei (Jadin and VercammenGrandjean), T. sicei (Andre).

Host: Fowl, mice, rats.

Distribution: Brundi, Central Africa, Ruanda.

T. uganda (Vercammen-Grandjean and Brennan).

Host: Dogs.

Distribution: Uganda.

T. centropodis (Ewing).

Host: Birds

Distribution: Liberia.

T. claviglicola (Lawrence).

Host: Mice.

Distribution: S. Africa.

Family: Xolagidae (Dubini)

1-Leptoshyra (Hull)

L. acuticubita (Gaud),

L. centropoda (Megnin).

Host: Birds.

Distribution: Cameroons.

L. arenariae (Gaud).

Host: Birds, ducks 